\title{
Asenapine for the treatment of adults with an acute exacerbation of schizophrenia: results from a randomized, double-blind, fixed-dose, placebo- controlled trial with olanzapine as an active control
}

\author{
Ronald Landbloom, 'Mary Mackle, 'Xiao Wu, Linda Kelly, 'Linda Snow-Adami, \\ Roger S. McIntyre, ${ }^{3,4 *}$ Maju Mathews, ${ }^{5}$ and Carla Hundt ${ }^{5}$
}

\footnotetext{
${ }^{1}$ Department of Neuroscience, Merck, Kenilworth, New Jersey, USA

${ }^{2}$ Department of Statistical Science, Allergan, Jersey City, New Jersey, USA

${ }^{3}$ Department of Psychiatry and Pharmacology, University of Toronto, Toronto, Ontario, Canada

${ }^{4}$ Mood Disorders Psychopharmacology Unit, University Health Network, Toronto, Ontario, Canada

${ }^{5}$ Department of Psychiatry, Forest Research Institute (now Allergan), Jersey City, New Jersey, USA
}

Objective. Evaluate the efficacy and safety of asenapine $2.5 \mathrm{mg}$ twice daily (bid; $\mathrm{n}=97$ ) or $5 \mathrm{mg}$ bid ( $\mathrm{n}=113$ ) versus placebo $(\mathrm{n}=101)$ in adults with acute exacerbation of schizophrenia.

Methods. Adults with Diagnostic and Statistical Manual of Mental Disorders, Fourth Edition, Text Revision (DSM-IV-TR) schizophrenia diagnosis were randomized to asenapine $2.5 \mathrm{mg}$ bid, $5 \mathrm{mg}$ bid, placebo, or olanzapine $15 \mathrm{mg}$ once daily. The primary objective was to test superiority of asenapine versus placebo as measured by the change from baseline to day 42 in the Positive and Negative Syndrome Scale (PANSS) total score. The key safety objective was to evaluate weight change in asenapine versus olanzapine at day 42 .

Results. The primary efficacy endpoint was met; the difference in least squares mean change from baseline to day 42 in PANSS total score between asenapine $5 \mathrm{mg}$ bid and placebo was -5.5 points (unadjusted $95 \%$ CI: -10.1 , -1.0 ; multiplicity adjusted $P=0.0356$ ). Neither asenapine $2.5 \mathrm{mg}$ bid nor olanzapine $15 \mathrm{mg}$ were superior to placebo.

Both asenapine groups demonstrated significantly less weight gain than olanzapine at day 42 . Significantly higher incidences of oral hypoesthesia and dysgeusia (combined) for asenapine $2.5 \mathrm{mg}$ bid $(5.2 \%$ vs $0.0 \% ; P=0.0217)$ and $5 \mathrm{mg}$ bid $(7.1 \%$ vs $0.0 \% ; P=0.0033)$ were observed versus placebo. There were no significant differences between asenapine and placebo for insomnia, extrapyramidal symptoms, akathisia, dizziness, or combination of somnolence/ sedation/hypersomnia.

Conclusion. This study supports previous efficacy and safety findings of asenapine; asenapine $5 \mathrm{mg}$ bid is the lowest effective dose in adults with schizophrenia. Asenapine was associated with significantly less weight gain than olanzapine at day 42 .

Received 16 October 2015; Accepted 25 March 2016; First published online 8 November 2016

Key words: Asenapine, efficacy, olanzapine, safety, schizophrenia.

\footnotetext{
* Address for correspondence: Roger S. McIntyre, MD, FRCPC, Department of Psychiatry and Pharmacology, University of Toronto, University Health Network, 399 Bathurst Street, MP 9-325, Toronto, ON M5T 2S8, Canada. (Email: roger.mcintyre@uhn.ca)

Data from this article have been presented previously at the meetings of the American Psychiatric Association (May 16 - 20, 2015, Toronto, Ontario) and the American Society of Clinical Psychopharmacology (June 22 - 26, 2015, Miami, Florida).

Tonya Goodman, of Arbor Communications, Inc., Ann Arbor, MI, provided editorial assistance in the preparation of this manuscript, funding for which was provided by Forest Laboratories, LLC, an Allergan affiliate.

Clinical trial registration: NCT01617187.
} 


\section{Introduction}

Schizophrenia affects approximately 26 million people worldwide $^{1}$ with diverse psychological processes that include both positive and negative symptoms. ${ }^{2,3}$ Most patients with schizophrenia suffer from recurrent exacerbations of positive symptoms as well as persistent negative and cognitive symptoms, all of which require lifetime disease management.

First-generation antipsychotics function through dopamine receptor blockade and primarily influence positive symptoms, while second-generation antipsychotics are also active at other receptor sites. ${ }^{3}$ This additional activity may enable second-generation antipsychotics to affect negative symptoms, ${ }^{3}$ although not all antipsychotics have demonstrated such ability. ${ }^{4,5}$ Furthermore, the adverseeffect profiles of these agents differ. First-generation antipsychotics may lead to extrapyramidal symptoms (EPS), such as dystonias, dyskinesia, and akathisia, whereas second-generation antipsychotics may be associated with sedation, weight gain, treatment-emergent diabetes mellitus, and agranulocytosis.

Asenapine, a sublingual second-generation atypical antipsychotic, is indicated for acute and maintenance treatment of schizophrenia in adults at $5 \mathrm{mg}$ twice daily (bid) or $10 \mathrm{mg}$ bid. ${ }^{6}$ Asenapine has a unique receptorbinding profile that displays potent multi-receptor antagonism for serotonin, dopamine, noradrenaline, and histamine receptors. ${ }^{7}$ Asenapine safety and efficacy were evaluated in short- and long-term studies. ${ }^{8-11}$ In previous trials, asenapine $5 \mathrm{mg}$ bid had an improved safety profile over asenapine $10 \mathrm{mg}$ bid. ${ }^{9,11}$ However, asenapine $5 \mathrm{mg}$ bid has not been compared with a lower dose in an active-comparator trial in schizophrenia patients. A phase 2 study evaluated lower doses of asenapine without an active comparator, thus providing the impetus for a post-approval commitment to evaluate safety and efficacy of a dose $<5 \mathrm{mg}$ bid in schizophrenia. We sought to evaluate the efficacy and safety of asenapine $2.5 \mathrm{mg}$ bid or $5 \mathrm{mg}$ bid relative to placebo in this population.

\section{Methods}

\section{Participants and study design}

This 6-week, randomized, double-blind, double-dummy, fixed-dose trial (NCT01617187) across 65 sites in 7 countries (22 in the United States, 10 in Bulgaria, 8 in Romania, 13 in the Russian Federation, 7 in Croatia, and 5 in Ukraine) from January 2013 to September 2014 evaluated the efficacy and safety of asenapine versus placebo in patients with acute exacerbation of schizophrenia. The protocol was approved by an Independent Ethics Committee or Institutional Review Board, and was conducted in compliance with the Declaration of Helsinki, International Conference on Harmonization Guidelines, and Good Clinical Practice. Patient written informed consent was obtained.

\section{Key inclusion criteria}

Patients were aged $\geq 18$ years with a current Diagnostic and Statistical Manual of Mental Disorders, Fourth Edition, Text Revision (DSM-IV-TR) diagnosis of schizophrenia of paranoid (295.30), disorganized (295.10), or undifferentiated subtype (295.90) with a Positive and Negative Syndrome Scale (PANSS) ${ }^{2}$ total score $\geq 70$ at screening and baseline. Patients were required to have a score $\geq 4$ on at least 2 items in the PANSS positive subscale. A Clinical Global Impression-Severity (CGI-S) score $\geq 4$ (moderately ill) confirmed experience of an acute exacerbation of schizophrenia (as evidenced by a duration of $\leq 8$ weeks), and need of increased medical attention to treat worsening positive symptoms. Prior to baseline, patients must have previously responded positively to an antipsychotic medication other than clozapine and tapered off all prohibited psychotropic medication (antipsychotics, antidepressants, and mood stabilizers).

\section{Key exclusion criteria}

Patients with uncontrolled, unstable, clinically significant medical conditions (renal, endocrine, hepatic, respiratory, cardiovascular, hematologic, immunologic, or cerebrovascular disease, or malignancy) that may interfere with evaluations; body mass index $<18.5$ or $>40.0 \mathrm{~kg} / \mathrm{m}^{2}$; evidence of clinically significant hepatic conditions (alanine aminotransferase [ALT] or aspartate aminotransferase [AST] $>3 \times$ the upper limit of normal [ULN] and total bilirubin $>2 \times \mathrm{ULN}$, or ALT or AST $>3 \times$ ULN with jaundice, worsening fatigue, nausea, vomiting, right upper quadrant pain or tenderness, fever, rash, or eosinophilia); clinically significant laboratory, vital sign, physical examination, or electrocardiogram findings at screening that precluded trial participation; narrow-angle glaucoma history; epilepsy or any seizure disorder beyond childhood febrile seizure diagnosis; human immunodeficiency virus antibody serological evidence; neuroleptic malignant syndrome history; or tardive dyskinesia history were excluded.

Additional exclusion criteria included diagnosis of schizoaffective disorder or schizophrenia of catatonic or residual subtype, or schizophrenia of residual subtype; primary Axis I diagnosis other than schizophrenia; comorbid Axis I disorder responsible for current symptoms and functional impairment; borderline personality disorder diagnosis; mental retardation diagnosis, history of traumatic brain injury causing ongoing cognitive 
difficulties, Alzheimer's disease or another form of dementia, or any central nervous system chronic organ disease; meeting DSM-IV-TR criteria for substance abuse/dependence (except nicotine) within the past 6 months; positive urine drug/alcohol screen; diagnosis of psychotic disorder or disturbance thought to be substance-induced; $>20 \%$ decrease in PANSS total score from screening to baseline; at imminent risk of self-harm or harm to others; imprisonment, parole, or assaultive behavior within the past 2 years; or under involuntary inpatient commitment due to being considered a danger to him/herself or others. Patients who received prior treatment with asenapine for the current episode, were previously nonresponsive or experienced an allergic reaction to asenapine, or were treated in a previous asenapine trial were excluded; patients could not have taken clozapine within 12 weeks before baseline for treatment-resistant schizophrenia.

\section{Procedures}

Patients were randomized 2:2:2:1 to asenapine $2.5 \mathrm{mg}$ bid, asenapine $5 \mathrm{mg}$ bid, placebo, or olanzapine $15 \mathrm{mg}$ once daily (qd) (Figure 1A). Evaluation of asenapine $2.5 \mathrm{mg}$ bid was requested as part of a post-approval commitment because this dose had demonstrated potential efficacy in a dose-finding study. Olanzapine efficacy ranges from 10 to $15 \mathrm{mg}$ qd per the product label. ${ }^{12}$ Therefore, the $15 \mathrm{mg}$ qd dose was selected as the active comparator $(10 \mathrm{mg}$ qd administered during week 1 ). In this trial, the active comparator was included to allow comparison of safety findings and to confirm assay sensitivity; no efficacy comparisons were made between active treatments.

The majority of patients $(63.3 \%)$ were inpatients at the time of enrollment. All patients (including outpatients at the time of enrollment) were hospitalized at screening and for the first 3 weeks of double-blind treatment. Patients who were clinically stable according to the investigator's judgment could be discharged at week 3 and continue the study as outpatients.

\section{Study outcomes}

The primary hypothesis of this study was that at least one of the asenapine doses (2.5 $\mathrm{mg}$ and $5 \mathrm{mg}$ bid) was superior to placebo in improving schizophrenia symptoms. The primary endpoint was the change from baseline to day 42 in PANSS total score. PANSS is a 30-item clinician-rated instrument for schizophrenia symptoms with each item rated from 1 (absent) to 7 (extreme). Key secondary efficacy endpoints included change from baseline to day 42 in CGI-S and rate of PANSS responders $(\geq 50 \%$ reduction in PANSS total score) at day 42 . CGI-S is a 7-point clinician-rated scale to assess illness severity. ${ }^{13,14}$

Safety and tolerability were monitored in patients who took $\geq 1$ dose of study medication (ie, all-treated set [ATS]). The key safety objective was weight change associated with asenapine versus olanzapine as measured by the mean change from baseline to day 42 . Other key safety events included insomnia, akathisia, combined somnolence/sedation/hypersomnia, dizziness, combined hypoesthesia/dysgeusia, and EPS.

Adverse events (AEs) were defined as any unfavorable or unintended change in the structure, function, or chemistry of the body temporally associated with use of sponsor product, whether or not considered related to the product. AEs were coded using MedDRA version 17.0. AEs were designated treatment-emergent (TEAEs) if they were newly reported or worsened in severity since baseline. EPS were documented with the Simpson Angus Rating Scale, Barnes Akathisia Rating Scale, and Abnormal Involuntary Movement Scale. ${ }^{15-19}$ EPS TEAEs were defined using the narrow standardized MedDRA query (SMQ), which included Parkinsonism, dyskinesia, akathisia, and dystonia.

Metabolic indices, laboratory tests, vital signs, physical examination findings, electrocardiogram results, and pregnancies were also evaluated. Closely monitored events included evidence of drug-induced liver damage, tardive dyskinesia, syncope, new-onset diabetes mellitus, emerging or increasing suicidal ideation and/or behavior, and drug hypersensitivity reactions.

\section{Sample size}

Assuming a $35 \%$ failure rate, screening 550 patients was planned with 354 randomized to provide $\geq 101$ patients each for placebo, asenapine $2.5 \mathrm{mg}$ bid, and asenapine $5 \mathrm{mg}$ bid groups, and 51 patients for olanzapine, assuming a standard deviation of 20 points in change from baseline in PANSS total score with conservative adjustment made using the Bonferroni approach. ${ }^{20}$ This sample size would enable detection, with $90 \%$ power at $5 \%$ significance using a 2-sided test, of a 10-point difference in mean change from baseline in PANSS total score between placebo and asenapine, and detection with $89 \%$ power at $5 \%$ significance using a 1 -sided test of the difference between placebo and olanzapine.

\section{Statistical analysis}

For patient allocation, a sponsor-created computergenerated randomization list (using SAS software [SAS Institute Inc., Cary, NC, USA]) was used and stratified by site with 2:2:2:1 ratio for asenapine $2.5 \mathrm{mg}$ bid, asenapine $5 \mathrm{mg}$ bid, placebo, and olanzapine. Patient 


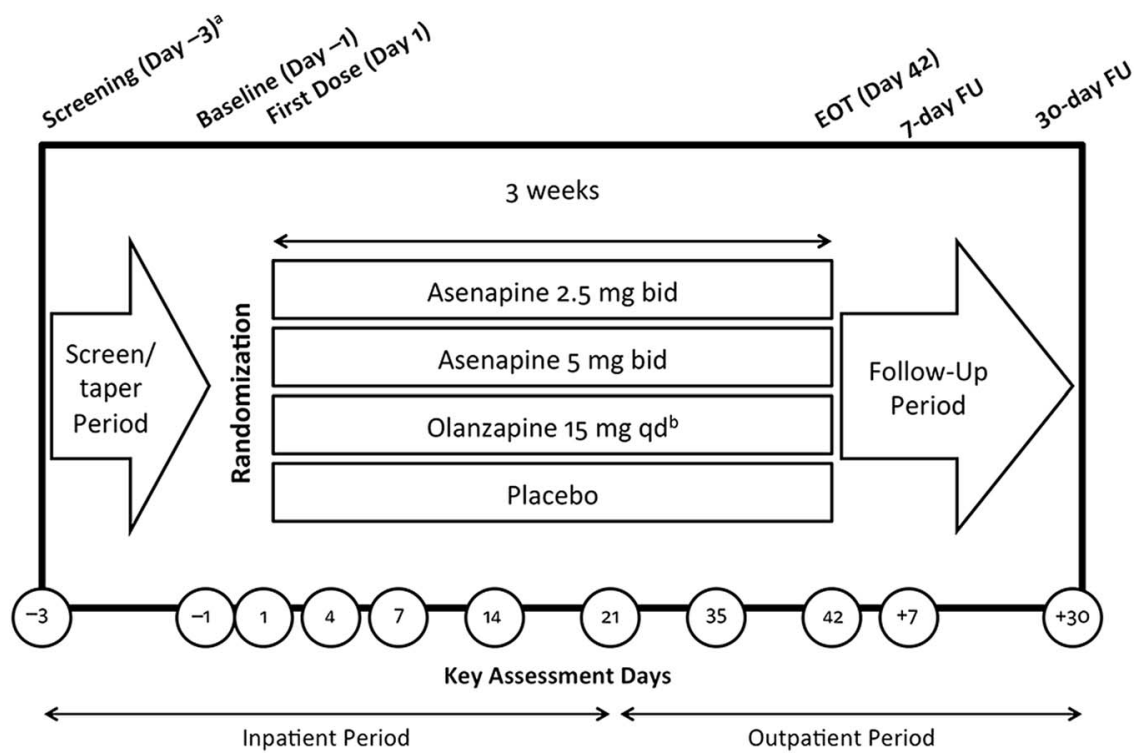

FIGURE 1A. Clinical study design. bid, twice daily; EOT, end of treatment; FU, follow-up; qd, once daily.

${ }^{a}$ The screening and tapering period could have been extended up to 7 days to allow time for discontinuation from prohibited medications.

bexcept during week 1, when $10 \mathrm{mg}$ was administered. The time of the active olanzapine dose (either a.m. or p.m.) was not disclosed to preserve blinding. The same numbers of film-coated tablets were taken in the a.m. and the p.m.

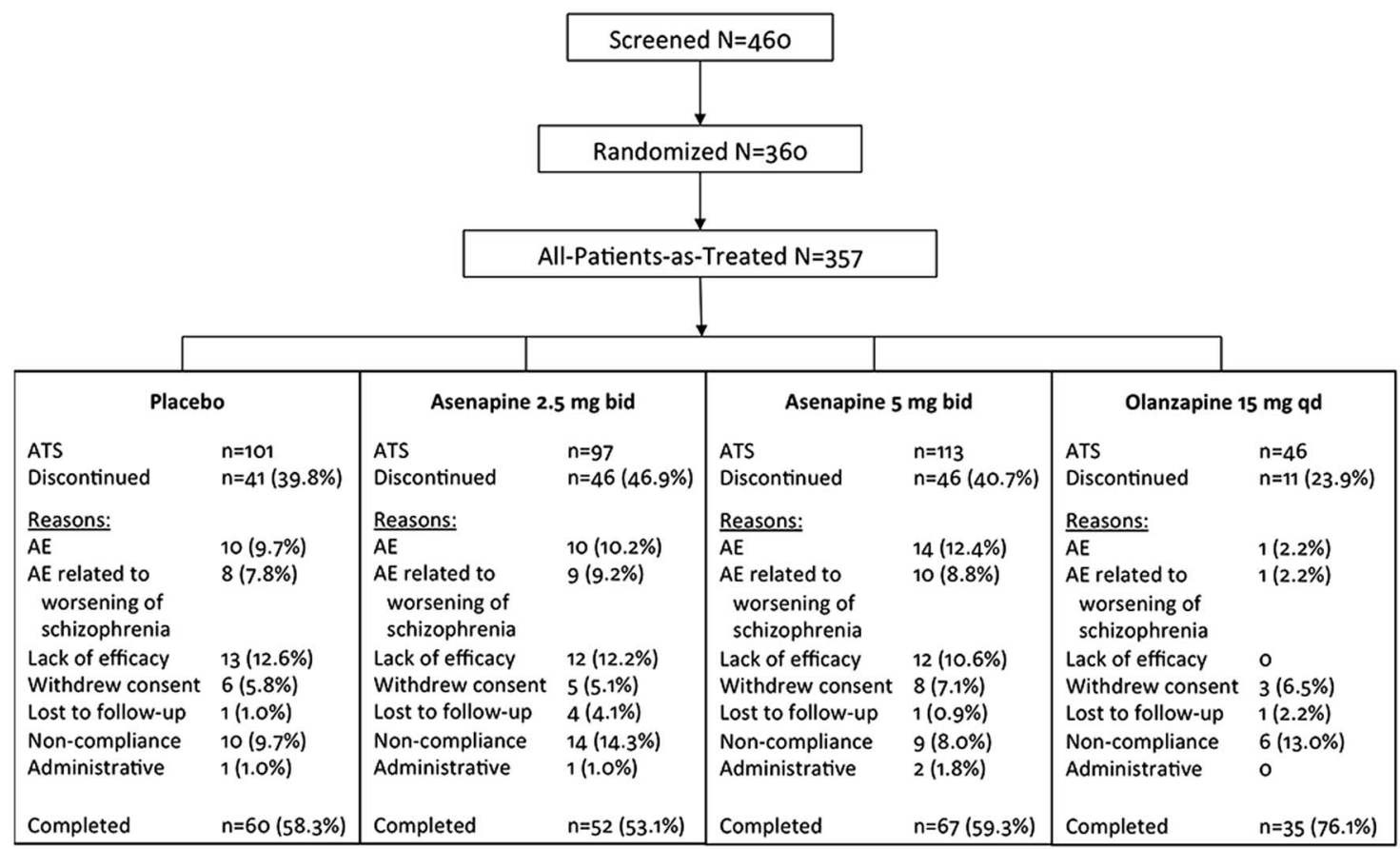

FIGURE 1B. Patient disposition. AE, adverse event; ATS, all-treated set; bid, twice daily; qd, once daily.

and medication allocation was performed by an independent third-party vendor.

Efficacy and body weight endpoints were analyzed using a mixed-effects model for repeated measurement (MMRM) with adjustment for multiple treatment comparisons using the full analysis set (FAS), defined as randomized patients who received $\geq 1$ dose of medication with $\geq 1$ baseline and post-baseline PANSS measurement. Statistical tests for each efficacy and body weight endpoint were conducted at an overall 2-sided 0.05 significance level. Multiplicity adjustment for 2 efficacy comparisons was made for primary and secondary endpoints. 


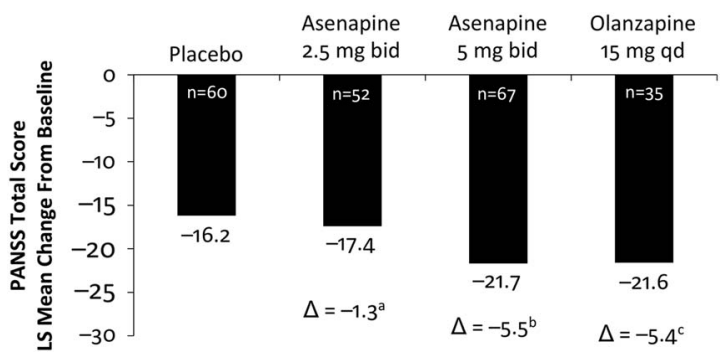

FIGURE 2. Least-squares mean difference in asenapine and olanzapine vs placebo in PANSS total score at day 42. bid, twice daily; $\Delta$, difference from placebo; PANSS, Positive and Negative Syndrome Scale; qd, once daily.

aUnadjusted $P=0.6043$, not significant.

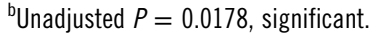

'Unadjusted $P=0.0587$, not significant.

Hochberg adjustments were applied to the 2 comparisons of body weight. ${ }^{21}$ Treatment comparisons were supported by $95 \%$ confidence intervals (CI) for the mean difference between groups. Efficacy and body weight endpoints were also analyzed using analysis of covariance (ANCOVA) to evaluate potential bias caused by missing data. Efficacy binary endpoints were analyzed using the Cochran Mantel-Haenszel test with an adjustment for investigative site. The rate of PANSS responders over time was analyzed using a generalized linear mixed model that assumed a binary distribution for response.

Safety analysis followed a multi-tiered approach. Predefined tier levels determined the rigorousness of statistical methods applied. Tier 1 included somnolence/ sedation/hypersomnia combined; dizziness; insomnia; oral hypoesthesia and dysgeusia; akathisia; EPS narrow SMQ; and $\geq 7 \%$ body weight increase. Tier 1 TEAEs were subject to statistical tests conducted at a 2-sided 0.05 significance level; $P$ values and $95 \%$ CIs for betweengroup comparisons were provided. Tier 2 events included those occurring in $\geq 4$ patients in any group; change from baseline to endpoint in metabolic parameters was assessed via point estimates with 95\% CIs provided for between-group comparisons. Point estimates by treatment group were provided for Tier 3 events, which included AEs occurring in $<4$ patients in all groups. The Miettinen and Nurminen method ${ }^{22}$ was used to perform $P$ value analysis (Tier 1 only) and $95 \%$ CIs (Tier 1 and Tier 2) for between-treatment differences. ANCOVA was used to obtain $P$ values (Tier 1 only) and $95 \%$ CIs (Tier 1 and Tier 2) for between-treatment differences.

\section{Results}

Of the 460 patients screened, 357 were treated with $\geq 1$ dose of study medication (Figure 1B). One asenapine $5 \mathrm{mg}$ bid-randomized patient was not treated owing to protocol noncompliance, and 2 placebo-randomized patients were not treated owing to administrative reasons. The FAS included 351 patients; 6 patients in the ATS were excluded from the FAS (placebo $=2$; asenapine $2.5 \mathrm{mg}$ bid $=1$; asenapine $5.0 \mathrm{mg}$ bid $=2$; olanzapine $15.0 \mathrm{mg} \mathrm{qd}=1$ ) as they did not have baseline or at least one post-baseline PANSS total score measurement. The most common reasons for discontinuation were protocol noncompliance, lack of efficacy, and AE. Baseline patient demographics were generally similar across treatment groups with the following exceptions: there was a slightly greater percentage of females in the placebo group compared with other groups, and there was a greater percentage of black patients in the olanzapine group compared with other groups (Supplemental Table 1, available online).

The primary endpoint was the change from baseline to day 42 in PANSS total score. The primary efficacy endpoint was met. The difference in least-squares (LS) mean change from baseline to day 42 in PANSS total score between asenapine $5 \mathrm{mg}$ bid and placebo was -5.5 points (unadjusted 95\% CI: $-10.1,-1.0$; multiplicity adjusted $P=0.0356$ ) (Figure 2, Table 1). Asenapine $2.5 \mathrm{mg}$ bid was not superior to placebo. An exploratory comparison of olanzapine to placebo at day 42 was suggestive of efficacy, with LS mean differences versus placebo of similar magnitude for olanzapine (-5.4 points) and asenapine $5 \mathrm{mg}$ bid ( -5.5 points); however, the difference between olanzapine and placebo was not statistically significant $(P=0.0587)$, likely due to the small sample size of the olanzapine group.

Key secondary endpoints were not met; neither asenapine dose was significantly superior to placebo in change from baseline to day 42 in CGI-S overall score (Table 1). Moreover, an exploratory comparison of olanzapine with placebo at day 42 did not show a statistically significant difference in this endpoint. Similarly, neither asenapine dose nor olanzapine was statistically significant compared with placebo in rate of PANSS responders at day 42 (Table 1). At day $42,19.2 \%, 14.6 \%, 25.2 \%$, and $26.7 \%$ of patients treated with placebo, asenapine $2.5 \mathrm{mg}$ bid, asenapine $5 \mathrm{mg}$ bid, and olanzapine, respectively, were considered PANSS responders (unadjusted $P=0.3700$, $P=0.1708$, and $P=0.2620$ for the difference between placebo and asenapine $2.5 \mathrm{mg}$ bid, asenapine $5 \mathrm{mg}$ bid, and olanzapine, respectively).

No deaths occurred during the study. Serious AEs were reported by $8(7.9 \%), 5(5.2 \%), 5(4.4 \%)$, and $1(2.2 \%)$ patients treated with placebo, asenapine $2.5 \mathrm{mg}$ bid, asenapine $5 \mathrm{mg}$ bid, and olanzapine, respectively (Table 2). The numbers of patients who discontinued treatment owing to AEs were similar between placebo and asenapine groups but lower in the olanzapine group (Table 2, Supplemental Table 2). Discontinuations owing to treatment-related AEs were similar. 
TABLE 1. Inferential analysis of efficacy objectives in the full analysis set (MMRM)

\begin{tabular}{|c|c|c|c|c|}
\hline Parameter & $\begin{array}{c}\text { PB0 } \\
n=99\end{array}$ & $\begin{array}{c}\text { ASN } \\
2.5 \mathrm{mg} \text { bid } \\
\mathrm{n}=96\end{array}$ & $\begin{array}{c}\text { ASN } \\
5 \mathrm{mg} \text { bid } \\
\mathrm{n}=111\end{array}$ & $\begin{array}{c}0 \mathrm{LZ} \\
15 \mathrm{mg} \text { qd } \\
\mathrm{n}=45\end{array}$ \\
\hline \multicolumn{5}{|l|}{ PANSS total score } \\
\hline $\mathrm{N}^{\mathrm{a}}$ & 60 & 52 & 67 & 35 \\
\hline LS mean change from baseline to day 42 (SE) & $-16.2(1.71)$ & $-17.4(1.80)$ & $-21.7(1.60)$ & $-21.6(2.32)$ \\
\hline LS mean difference (SE) in treatment vs PBO & & $-1.3(2.46)$ & $-5.5(2.32)$ & $-5.4(2.86)$ \\
\hline $95 \% \mathrm{Cl}$ & & $-6.1,3.6$ & $-10.1,-1.0$ & $-11.1,0.2$ \\
\hline$P$ value & & 0.6043 & 0.0178 & 0.0587 \\
\hline$P$ value, adjusted ${ }^{b}$ & & 0.6043 & 0.0356 & \\
\hline \multicolumn{5}{|l|}{ CGI-S } \\
\hline $\mathrm{N}^{\mathrm{a}}$ & 58 & 52 & 67 & 35 \\
\hline LS mean change from baseline to day 42 (SE) & $-1.0(0.11)$ & $-0.9(0.11)$ & $-1.2(0.10)$ & $-1.1(0.14)$ \\
\hline LS mean difference (SE) in treatment vs PBO & & $0.0(0.15)$ & $-0.3(0.14)$ & $-0.2(0.18)$ \\
\hline $95 \% \mathrm{Cl}$ & & $-0.3,0.3$ & $-0.6,0.0$ & $-0.5,0.2$ \\
\hline$P$ value & & 0.9083 & 0.0601 & 0.3898 \\
\hline Rate of PANSS responders ${ }^{c}$ & $19.2 \%$ & $14.6 \%$ & $25.2 \%$ & $26.7 \%$ \\
\hline$P$ value & & 0.3700 & 0.1708 & 0.2620 \\
\hline
\end{tabular}

\section{TABLE 2. Analysis of safety events in the treatment phase of the all-treated set}

\begin{tabular}{|c|c|c|c|c|}
\hline Category & $\begin{array}{c}\text { PB0 } \\
n=101\end{array}$ & $\begin{array}{c}\text { ASN } \\
2.5 \mathrm{mg} \text { bid } \\
\mathrm{n}=97\end{array}$ & $\begin{array}{c}\text { ASN } \\
5 \mathrm{mg} \text { bid } \\
n=113\end{array}$ & $\begin{array}{c}0 \mathrm{LZ} \\
15 \mathrm{mg} \text { qd } \\
\mathrm{n}=46\end{array}$ \\
\hline \multicolumn{5}{|l|}{ Patients reporting any $A E, n(\%)$} \\
\hline$\geq 1 \mathrm{AE}$ & $62(61.4)$ & $58(59.8)$ & $71(62.8)$ & $25(54.3)$ \\
\hline$\geq 1 \mathrm{TEAE}$ & $59(58.4)$ & $54(55.7)$ & $68(60.2)$ & $23(50.0)$ \\
\hline$\geq 1$ treatment-related TEAE & $28(27.7)$ & $25(25.8)$ & $38(33.6)$ & $17(37.0)$ \\
\hline$\geq 1$ severe TEAEs & $4(4.0)$ & $6(6.2)$ & $5(4.4)$ & $1(2.2)$ \\
\hline$\geq 1$ SAE & $8(7.9)$ & $5(5.2)$ & $5(4.4)$ & $1(2.2)$ \\
\hline$\geq 1$ treatment-related SAEs & $1(1.0)$ & $1(1.0)$ & $0(0.0)$ & $0(0.0)$ \\
\hline$\geq 1 \mathrm{AE}$ leading to treatment discontinuation & $10(9.9)$ & $10(10.3)$ & $14(12.4)$ & $1(2.2)$ \\
\hline$\geq 1$ treatment-related $A E$ leading to treatment discontinuation & $4(4.0)$ & $5(5.2)$ & $7(6.2)$ & $0(0.0)$ \\
\hline$\geq 1 \mathrm{AE}$ leading to death & $0(0.0)$ & $0(0.0)$ & $0(0.0)$ & $0(0.0)$ \\
\hline \multicolumn{5}{|c|}{ TEAE $\geq 5 \%$ in any asenapine group and $>2$ times placebo in the all-treated set, $n(\%)$} \\
\hline Patients with $\geq 1$ TEAE & $4(4.0)$ & $18(18.6)$ & $27(23.9)$ & $9(19.6)$ \\
\hline Nervous system disorders & $2(2.0)$ & $12(12.4)$ & $17(15.0)$ & $8(17.4)$ \\
\hline Headache & $2(2.0)$ & $10(10.3)$ & $10(8.8)$ & $4(8.7)$ \\
\hline Somnolence & $0(0.0)$ & $3(3.1)$ & $7(6.2)$ & $5(10.9)$ \\
\hline Gastrointestinal disorders & $0(0.0)$ & $4(4.1)$ & $7(6.2)$ & $0(0.0)$ \\
\hline Hypoesthesia oral & $0(0.0)$ & $4(4.1)$ & $7(6.2)$ & $0(0.0)$ \\
\hline Psychiatric disorders & $2(2.0)$ & $5(5.2)$ & $2(1.8)$ & $1(2.2)$ \\
\hline Psychotic disorder & $2(2.0)$ & $5(5.2)$ & $2(1.8)$ & $1(2.2)$ \\
\hline Investigations & $0(0.0)$ & $0(0.0)$ & $6(5.3)$ & $0(0.0)$ \\
\hline Blood creatine phosphokinase increased & $0(0.0)$ & $0(0.0)$ & $6(5.3)$ & $0(0.0)$ \\
\hline
\end{tabular}

AE, adverse event; ASN, asenapine; bid, twice daily; OLZ, olanzapine; PBO, placebo; qd, once daily; SAE, serious adverse event; TEAE, treatment-emergent adverse event. Adverse events were coded using the Medical Dictionary for Regulatory and Medical Affairs (MedDRA) dictionary version 17.0. The denominator for percentages is the number of patients in each treatment group. TEAEs are adverse events first reported or worsened in severity on or after the first dose of double-blind study drug through one of the following: last dose +7 days (adverse events) or last dose +30 days (serious adverse events) for patients who discontinue early or do not enter the extension trial; or the day prior to first dose date in the extension trial for patients continuing (applies to adverse events and serious adverse events). Patients were counted only once within each system organ class and preferred term. 
TABLE 3. Predefined treatment-emergent adverse events (TEAE) of special interest in the all-treated set

\begin{tabular}{|c|c|c|c|c|}
\hline & $\begin{array}{c}\text { PBO } \\
\mathrm{n}=101\end{array}$ & $\begin{array}{c}\text { ASN } \\
2.5 \mathrm{mg} \text { bid } \\
\mathrm{n}=97\end{array}$ & $\begin{array}{c}\text { ASN } \\
5 \mathrm{mg} \text { bid } \\
n=113\end{array}$ & $\begin{array}{c}0 \mathrm{LZ} \\
15 \mathrm{mg} \text { qd } \\
\mathrm{n}=46\end{array}$ \\
\hline Insomnia, n (\%) & $14(13.9)$ & $9(9.3)$ & $17(15.0)$ & $4(8.7)$ \\
\hline \% difference in treatment vs $\mathrm{PBO}(95 \% \mathrm{Cl})^{\mathrm{b}}$ & & $-4.6(-13.9,4.6)$ & $1.2(-8.6,10.8)$ & $-5.2(-15.2,7.7)$ \\
\hline$p^{\mathrm{b}}$ & & 0.3114 & 0.8057 & 0.3381 \\
\hline EPS SMQ (narrow), n (\%) & $7(6.9)$ & $5(5.2)$ & $7(6.2)$ & $2(4.3)$ \\
\hline \% difference in treatment vs $\mathrm{PBO}(95 \% \mathrm{Cl})$ & & $-1.8(-9.2,5.5)$ & $-0.7(-8.2,6.3)$ & $-2.6(-10.2,8.2)$ \\
\hline$P$ & & 0.5993 & 0.8284 & 0.5108 \\
\hline Somnolence, sedation, and hypersomnia combined, $\mathrm{n}(\%)$ & $3(3.0)$ & $3(3.1)$ & $9(8.0)$ & $5(10.9)$ \\
\hline \% difference in treatment vs $\mathrm{PBO}(95 \% \mathrm{Cl})$ & & $0.1(-5.7,6.1)$ & $5.0(-1.4,11.9)$ & $7.9(-0.0,20.4)$ \\
\hline$P$ & & 0.9599 & 0.1022 & 0.1062 \\
\hline Akathisia, n (\%) & $5(5.0)$ & $3(3.1)$ & $5(4.4)$ & $1(2.2)$ \\
\hline \% difference in treatment vs $\mathrm{PBO}(95 \% \mathrm{Cl})$ & & $-1.9(-8.4,4.4)$ & $-0.5(-7.2,5.7)$ & $-2.8(-9.4,6.8)$ \\
\hline$P$ & & 0.5045 & 0.8561 & 0.3621 \\
\hline Hypoesthesia oral and dysgeusia combined, $n(\%)$ & $0(0.0)$ & $5(5.2)$ & $8(7.1)$ & $0(0.0)$ \\
\hline$\%$ difference in treatment vs PBO $(95 \% \mathrm{Cl})$ & & $5.2(1.4,11.5)$ & $7.1(3.3,13.4)$ & N/A \\
\hline$P$ & & 0.0217 & 0.0033 & N/A \\
\hline Dizziness, $n(\%)$ & $1(1.0)$ & $1(1.0)$ & $2(1.8)$ & $0(0.0)$ \\
\hline \% difference in treatment vs $\mathrm{PBO}(95 \% \mathrm{Cl})$ & & $0.0(-4.5,4.7)$ & $0.8(-3.8,5.4)$ & $-1.0(-5.4,6.8)$ \\
\hline$P$ & & 0.9771 & 0.6225 & 0.3149 \\
\hline \multicolumn{5}{|c|}{$\begin{array}{l}\text { ASN, asenapine; bid, twice daily; Cl, confidence interval; EPS, extrapyramidal symptoms; N/A, not applicable; OLZ, olanzapine; PBO, placebo; } \\
\text { qd, once daily; SMQ, standardized Medical Dictionary for Regulatory and Medical Affairs (MedDRA) query. } \\
\text { a TEAEs are adverse events first reported or worsened in severity on or after the first dose of double-blind study drug through one of the following: last } \\
\text { dose + } 7 \text { days (adverse events) or last dose }+30 \text { days (serious adverse events) for patients who discontinue early or do not enter into the extension trial; } \\
\text { or the day prior to the first dose date in the extension trial for patients continuing (applies to both adverse events and serious adverse events). } \\
{ }^{\mathrm{b}} \text { The Miettinen and Nurminen method was used to perform the analysis for the } P \text { values and } 95 \% \text { Cls for between-treatment differences in the } \\
\text { percentage of patients with events. }\end{array}$} \\
\hline
\end{tabular}

Most TEAEs were considered by the investigator to be mild or moderate in intensity. A dose-dependent relationship in the incidence of TEAEs in asenapine groups appeared; however, TEAE incidences were similar between placebo and asenapine groups and lower for olanzapine (Table 2). The incidence of TEAEs was $58.4 \%, 55.7 \%, 60.2 \%$, and $50.0 \%$ for placebo, asenapine $2.5 \mathrm{mg}$ bid, asenapine $5 \mathrm{mg}$ bid, and olanzapine, respectively. Headache, somnolence, oral hypoesthesia, psychotic disorder, and increased blood creatinine phosphokinase occurred at an incidence $\geq 5 \%$ in at least 1 asenapine group and more than twice that of placebo (Table 2). Insomnia, somnolence, oral hypoesthesia, and increased blood creatine phosphokinase levels appeared to be potentially asenapine dose-dependent.

The key safety hypothesis was met; both asenapine groups showed significantly less weight gain than olanzapine at day 42 . The difference in LS mean change from baseline to day 42 in body weight was $-1.2 \mathrm{~kg}$ (unadjusted 95\% CI: $-2.4,-0.0$; multiplicity adjusted $P=0.0491$ ) between asenapine $2.5 \mathrm{mg}$ bid and olanzapine and $-1.2 \mathrm{~kg}$ (unadjusted 95\% CI: $-2.3,-0.0$; multiplicity adjusted $P=0.0491$ ) between asenapine $5 \mathrm{mg}$ bid and olanzapine. Sensitivity analysis suggested a difference between asenapine $2.5 \mathrm{mg}$ bid and olanzapine but not between asenapine $5 \mathrm{mg}$ bid and olanzapine at day 42 . There was no significant difference in patients with $\geq 7 \%$ weight gain between either asenapine group (asenapine $2.5 \mathrm{mg}$ bid, $4.3 \% ; 5 \mathrm{mg}$ bid, $8.5 \%)$ and placebo $(4.1 \%)$ or between olanzapine (13.0\%) and placebo (all $P>0.09$, not significant).

A significantly higher incidence of oral hypoesthesia and dysgeusia (combined) for asenapine $2.5 \mathrm{mg}$ bid $(5.2 \%$ vs $0.0 \% ; P=0.0217)$ and asenapine $5 \mathrm{mg}$ bid ( $7.1 \%$ vs $0.0 \% ; P=0.0033$ ) versus placebo was observed (Table 3$)$. There were no statistically significant differences between asenapine groups and placebo, or between olanzapine and placebo, for insomnia, EPS, akathisia, dizziness, or the combination of somnolence, sedation, and hypersomnia.

With regard to endocrine parameters, statistical results suggested differentiation for prolactin levels in both asenapine groups and olanzapine versus placebo (with smaller decreases from baseline seen in the asenapine groups compared with the placebo group; Supplemental Table 3). For metabolic parameters, differentiation was suggested for fasting triglyceride levels in the olanzapine group (with a small mean increase from baseline). Differentiation was not suggested for insulin levels, glucose, HbA1c, or total cholesterol for asenapine versus placebo, with similar 
results for olanzapine. The proportion of patients with laboratory values outside the predefined limits of change (PDLC) was generally small and comparable across treatment groups (Supplemental Table 4). Similarly, no clinically meaningful changes in EPS were observed (Supplemental Table 5).

\section{Discussion}

The primary hypothesis was met; asenapine $5 \mathrm{mg}$ bid was statistically superior to placebo $(P=0.0178$ vs placebo) in the LS mean difference from baseline to day 42 in PANSS total score. Although the observed reduction from baseline was greater for asenapine $2.5 \mathrm{mg}$ bid than placebo, statistical superiority was not met $(P=0.6043)$. An exploratory comparison of the LS mean difference in PANSS total score of olanzapine and placebo at day 42 was not significant $(P=0.0587)$, although the magnitude of reduction was comparable with asenapine $5 \mathrm{mg}$ bid and numerically greater than placebo. This may be due to the small number of patients in the olanzapine arm as a consequence of the randomization scheme and sample size determination. No significant difference was observed between either asenapine or olanzapine treatment groups and placebo for the change in CGI-S from baseline to day 42 or in PANSS responders.

The key safety objective was met; both asenapine groups showed significantly less weight gain than olanzapine at day 42. A post-hoc analysis of 17 placeboand/or olanzapine-controlled asenapine trials (13 schizophrenia and 4 bipolar mania) demonstrated that asenapine was associated with an increased incidence of weight gain versus placebo but decreased incidence versus olanzapine with respect to triglycerides, weight gain, and elevated cholesterol. ${ }^{23}$ This study supports these findings.

The combination of oral hypoesthesia and dysgeusia occurred at a statistically significantly higher rate in asenapine groups versus placebo or olanzapine groups. There was no significant increase in the incidence of insomnia; EPS; somnolence, sedation, and hypersomnia combined; akathisia; or dizziness between asenapine groups and placebo or between olanzapine and placebo. No asenapine-treated patient had a TEAE of tardive dyskinesia, syncope, orthostatic hypotension, new onset diabetes mellitus, or hypersensitivity.

This study supports the efficacy and tolerability profiles of asenapine $5 \mathrm{mg}$ bid observed previously. ${ }^{8-10}$ Asenapine $5 \mathrm{mg}$ bid has previously demonstrated significant superiority over placebo in improving CGI-S. ${ }^{8-10}$ In the present study, asenapine $5 \mathrm{mg}$ bid failed to reach statistical superiority over placebo in CGI-S score, although results were numerically improved for asenapine versus placebo and olanzapine. Although there may be a correlation between clinical study results and results in a clinical setting, there is also a separation of clinical metrics.

These results may not be generalizable to the entire adult population with schizophrenia, particularly owing to the strict enrollment criteria used and the relative heterogeneity within this patient population. The shortterm nature of the trial does not permit long-term efficacy and safety conclusions to be drawn, particularly with respect to asenapine $2.5 \mathrm{mg}$ bid data.

It is also worthwhile to note that the relatively high discontinuation rates observed for the placebo and asenapine groups are consistent with what has been observed in previous asenapine placebo-controlled clinical trials. ${ }^{8-10}$ The discontinuation rate in the olanzapine group was lower than that in the asenapine and placebo groups; these findings are consistent with previous reports which have found lower discontinuation rates with olanzapine than with other atypical antipsychotics. ${ }^{24,25}$ Although olanzapine did not show statistically significant differences versus placebo on the primary efficacy parameter, the comparatively low discontinuation rate may be indicative of treatment response. ${ }^{25}$

\section{Conclusions}

Asenapine $5 \mathrm{mg}$ bid is proven to be the lowest effective dose in adults with schizophrenia based on short-term studies. 8,9 This study supports these findings along with the previously established safety profile of asenapine in adults with schizophrenia; no new or emerging safety signals were observed. The combination of oral hypoesthesia and dysgeusia occurred at a statistically significantly higher rate in the asenapine groups than in the placebo or olanzapine groups. Increases in body weight from baseline to day 42 were lower with asenapine than olanzapine.

\section{Disclosures}

Ronald Landbloom, Mary Mackle, Linda Kelly, and Linda Snow-Adami are employees of Merck \& Co., and may own stock or have stock options. Renee Wu is an employee of Allergan and may own stock or have stock options. Roger S. McIntyre has been an advisory board member of Bristol-Myers Squibb, Eli Lilly, Lundbeck, Pfizer, Shire, Merck, Sunovion, Otsuka, Forest, and Takeda; received speakers fees from Janssen-Ortho, Astra-Zeneca, Eli Lilly, Lundbeck, Merck, Pfizer, Otsuka, Sunovion, Forest, and Takeda; and received research grants from Eli Lilly, Janssen-Ortho, Shire, Astra-Zeneca, Pfizer, Lundbeck, Forest, Sunovion, and BMS. Maju Mathews and Carla Hundt were employed at the time of the study by Forest Research Institute, an Allergan affiliate, and may own stock or have stock options. 
The study was designed by Merck \& Co., Inc., which had direct oversight or participation in every stage of the study. All authors had full access to the data after study completion and unblinding and are responsible for the work described in this paper. All authors were involved in at least 1 of the following: conception, design, acquisition, analysis, interpretation of data, and drafting the manuscript and/or revising the manuscript for important intellectual content. All authors provided final approval of the version to be published. Funding for the manuscript was provided by Forest Research Institute, an affiliate of Allergan, Inc.

\section{Supplementary material}

To view supplementary material for this article, please visit http://dx.doi.org/10.1017/S1092852916000377

\section{REFERENCES:}

1. Lora A, Kohn R, Levav I, McBain R, Morris J, Saxena S. Service availability and utilization and treatment gap for schizophrenic disorders: a survey in 50 low- and middle-income countries. Bulletin of the World Health Organization. 2012; 90(1): 47-54B.

2. Kay SR, Fiszbein A, Opler LA. The positive and negative syndrome scale (PANSS) for schizophrenia. Schizophr Bull. 1987; 13(2): 261-276.

3. Freedman R. Schizophrenia. $N$ Engl J Med. 2003; 349: 1738-1749.

4. Smith T WC, Lieberman J. Schizophrenia (maintenance treatment). Am Fam Physician. 2010; 82(4): 338-339.

5. Tandon R, Keshavan MS, Nasrallah HA. Schizophrenia, "just the facts": what we know in 2008, part 1: overview. Schizophr Res. 2008 , 100(1-3): 4-19.

6. SAPHRIS [package insert]. St. Louis, MO: Forest Pharmaceuticals, Inc.; 2015.

7. Shahid M, Walker GB, Zorn SH, Wong EH. Asenapine: a novel psychopharmacologic agent with a unique human receptor signature. J Psychopharmacol. 2009; 23(1): 65-73.

8. Potkin SG, Cohen M, Panagides J. Efficacy and tolerability of asenapine in acute schizophrenia: a placebo- and risperidonecontrolled trial. J Clin Psychiatry. 2007; 68(10): 1492-1500.

9. Kane JM, Cohen M, Zhao J, Alphs L, Panagides J. Efficacy and safety of asenapine in a placebo- and haloperidol-controlled trial in patients with acute exacerbation of schizophrenia. J Clin Psychopharmacol. 2010; 30(2): 106-115.
10. Kane JM, Mackle M, Snow-Adami L, Zhao J, Szegedi A, Panagides J. A randomized placebo-controlled trial of asenapine for the prevention of relapse of schizophrenia after long-term treatment. J Clin Psychiatry. 2011; 72(3): 349-355.

11. Schoemaker J, Naber D, Vrijland P, Panagides J, Emsley R. Long-term assessment of asenapine vs olanzapine in patients with schizophrenia or schizoaffective disorder. Pharmacopsychiatry. $2010 ;$ 43(4): 138-146.

12. Zyprexa [package insert]. Indianapolis, IN: Eli Lilly and Company; 1997.

13. Guy W. ECDEU Assessment Manual for Psychopharmacology Rev ed. Rockville, MD: National Institutes of Mental Health; 1976.

14. Spearing MK, Post RM, Leverich GS, Brandt D, Nolen W. Modification of the Clinical Global Impressions (CGI) Scale for use in bipolar illness (BP): the CGI-BP. Psychiatry Res. 1997; 73(3): 159-171.

15. Simpson GM, Angus JW. A rating scale for extrapyramidal side effects. Acta Psychiatr Scand Suppl. 1970; 212: 11-19.

16. Simpson GM, et al. Adverse effect measures. In: American Psychiatric Association. Handbook of Psychiatric Measures. Washington, DC: American Psychiatric Association; 2000: 163-164.

17. Barnes TR. A rating scale for drug-induced akathisia. $\mathrm{Br}$ J Psychiatry. 1989; 154(5): 672-676.

18. Barnes T. Adverse effect measures. In: American Psychiatric Association. Handbook of Psychiatric Measures. Washington, DC: American Psychiatric Association; 2000: 165-166.

19. Guy W. Abnormal involuntary movement scale (AIMS). In: Guy W. ECDEU Assessment Manual for Psychopharmacology. Revised 1976. Rockville, MD: National Institutes of Mental Health; 534-537.

20. Dunn OJ. Multiple comparisons among means. J Am Stat Assoc. 1961; 56(293): 52-64.

21. Hochberg Y. A sharper Bonferroni procedure for multiple tests of significance. Biometrika. 1988; 75(4): 800-802.

22. Mietinnen O, Nurminen M. Comparative analysis of two rates. Stat Med. $1985 ;$ 4(2): 213-226.

23. Kemp DE, Zhao J, Cazorla $\mathrm{P}$, et al. Weight change and metabolic effects of asenapine in patients with schizophrenia and bipolar disorder. J Clin Psychiatry. 2014; 75(3): 238-245.

24. McEvoy JP, Lieberman JA, Stroup TS, et al. Effectiveness of clozapine versus olanzapine, quetiapine, and risperidone in patients with chronic schizophrenia who did not respond to prior atypical antipsychotic treatment. Am J Psychiatry. 2006;163(4):600-610.

25. Kinon BJ, Liu-Seifert H, Adams DH, Citrome L. Differential rates of treatment discontinuation in clinical trials as a measure of treatment effectiveness for olanzapine and comparator atypical antipsychotics for schizophrenia. J Clin Psychopharmacol. $2006 ; 26(6): 632-637$. 